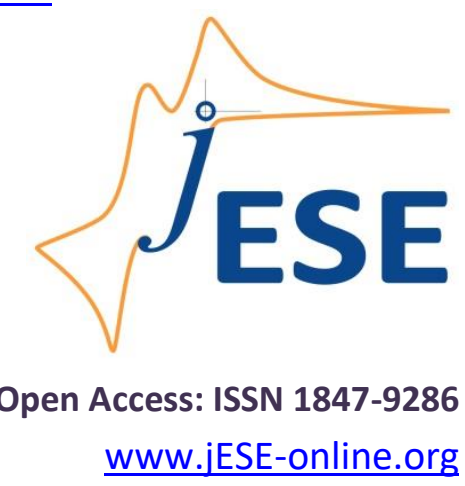

Original scientific paper

\title{
On the processes of migration and diffusion in the systems with solid-state reagents
}

\author{
Viacheslav Barsukov ${ }^{凶}$, Volodymyr Khomenko, Oksana Chernysh \\ Department for Electrochemical Power Engineering \& Chemistry, Kyiv National University of \\ Technologies and Design, 2 Nemyrovych-Danchenko str., Kyiv 01011, Ukraine \\ Corresponding author: ${ }^{\bowtie}$-barsukov@i.ua; Tel.: +380 -442-562-102; Fax: +380-442-848-266 \\ Received: November 14, 2019; Revised: January 10, 2020; Accepted: January 14, 2020
}

\begin{abstract}
This paper deals with peculiarities of diffusion and migration in electrochemical systems with solid-state reagents (ESSSR). Contradictions of the diffusion model are analyzed. It is the difference of applied potentials and the corresponding electric field strength in the bulk solid phase and at the interfaces which is the primary driving force of charge transfer in ESSSR. The time characteristic of diffusion processes is not comparable to the duration of electrode processes at charging/discharging of batteries and especially electrochemical capacitors. In many real systems involving ESSSR, the process of diffusion in solid phase is absent. Examples of charge transfer processes in ESSSR (nickel hydroxide electrode, sparingly soluble quinoid compounds, $\mathrm{Li}^{+}$intercalation in graphite, etc.) are considered, and the processes are explained using the Grothuss, tunnel and other migration mechanisms. It is shown in this paper that the linear relationship between peak currents in voltammetric curves and the square root of potential scan rate cannot be presented as an ultimate support of the diffusion model, but as a more universal property of ESSSR. In this aspect, the efficient diffusion coefficient, $D_{\text {eff, }}$ could be at best discussed, not to distort the ideas of charge-transfer migration mechanisms in the ESSSR.
\end{abstract}

\section{Keywords}

Solid-state electrochemical systems; charge transfer models; mechanisms; macrokinetics.

\section{Introduction}

Solid-state insoluble and sparingly soluble reagents are common in electrochemical practice and are widely used as anode and cathode materials in many electrochemical power sources (EPS), as insoluble anodes in electroplating, in corrosion protection systems, for electrochemical analyses, in electrochemical sensors and other fields of modern electrochemical science and engineering.

However, the theory of charge transfer processes in solid-state systems is developed insufficiently and is based mainly on the classical theoretical assumptions formulated essentially for solid- 
state reactions at the "electrolyte solution-solid phase" interface. In this context, the ideas about the diffusion of protons (or ions of lithium and sodium) in solid state, occurring for example in active materials of EPS electrodes, can be frequently found in modern electrochemical literature [1-7]. Moreover, it is usually believed a priori that it is just diffusion of cations in solid state that retards the discharge (charge) of electrodes. Then, an attempt was made to calculate the coefficient of diffusion of protons (or ions of lithium and sodium) in solid state, $D$, based on experimental data, under the assumption that the following Fick diffusion equation can be applied to experimental conditions [6]:

$$
j=-D \operatorname{grad} C
$$

In eq. (1), $j$ is the amount of material transported through unit area per second, while grad $C$ is the gradient of ions concentration. It is supposed by Fick diffusion equation (1) that the coefficient of diffusion $D$ is a constant value for the selected material and fixed temperature.

On the other hand, the current density is expressed as

$$
i=\sigma \operatorname{grad} E
$$

where $\sigma$ is conductivity of material, while grad $E$ is gradient of potentials.

It is necessary to note that $D$ values for some solid-state electrochemical systems that were already reported by different researchers and determined using different methods, commonly range over several orders of magnitude. For example, the attempts to determine $D$ value of protons in the solid phase of nickel hydroxide electrode (NHE) using the above mentioned approach, led to $D$ values ranging from $10^{-5}$ to $10^{-15} \ldots 10^{-16} \mathrm{~cm}^{2} / \mathrm{s}$ as it was shown by $\mathrm{V}$. A. Volynskij in [8,9] and especially in [8]. Obviously, such evaluation with differences of 10-11 orders of magnitude indicates that the diffusion model applied to NHE is inappropriate.

Our earlier studies (for example [10]) showed that models should take into account the experimentally proved presence of interlayer water stabilized by the layered structure of nickel (II, III) hydroxides in the grain of NHE. Then, the basically different mechanism of proton migration in the NHE grain, in particular, the Grothuss mechanism of proton migration in the interlayer water becomes possible in accordance with following equations:

$$
\mathrm{NiOOH}+\mathrm{H}_{2} \mathrm{O}+\mathrm{e}^{-} \leftrightarrow \mathrm{Ni}(\mathrm{OH})_{2}+\mathrm{OH}^{-} \text {or } \mathrm{NiOOH}+\mathrm{H}^{+}+\mathrm{e}^{-} \leftrightarrow \mathrm{Ni}(\mathrm{OH})_{2}
$$

The migration model of NHE grain which was developed in $[10,11]$, enables to explain the following main peculiarities of NHE behavior: reason for electrode passivation during the discharge; formally $1 \mathrm{e}^{-}$process at NHE; practically possible range of NHE cycling (up to 1.5-2.0 e) depending on the electrode morphology and construction; influence of contaminants and activating additives (especially, Co hydroxide) [12].

A similar "proton" mechanism is typical for sparingly soluble quinoid compounds, including anthraquinone-9,10 and its derivatives, p-chloranil, pentacenequinone-13,14, and some other representatives of this interesting class of materials. The reaction in such sparingly soluble electrochemically active compounds can be at the example of $p$-chloranil, described as follows:

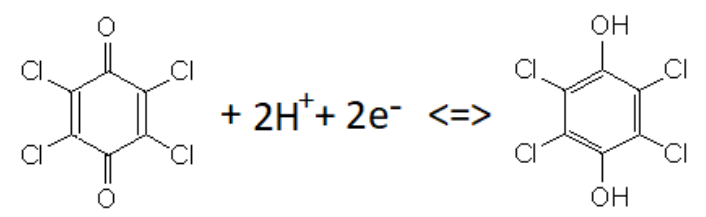

A proper understanding of the charge transfer mechanism in solid quinones is hindered not only by the extremely low coefficient of proton diffusion, but also very low conductivity. It has already been reported $[13,14]$ that quantum-mechanical tunneling is the most probable mechanism of 
hydrogen penetration through the potential barrier. The driving force of the process is the electric field strength in the solid phase, which distribution in the solid phase has a crucial effect on the macrokinetics of the process in the quinone grain.

Another example can be intercalation-deintercalation of $\mathrm{Li}^{+}$into graphite-carbon materials. Despite obvious difficulties in modelling, the processes of intercalation-deintercalation of lithium cations into the anode or cathode material, are associated with different conditions of their movement in the solid phase and at solid-electrolyte interface (SEI). Therefore, it is difficult to imagine that such processes can be described by simple diffusion models. Nevertheless, some authors used Fick equation to calculate the diffusion coefficient.

As it was shown by A. V. Churikov in [15] and especially [16], the scatter of $D$ values is again 10 orders, from $10^{-5}$ to $10^{-15} \mathrm{~cm}^{2} / \mathrm{s}$ for lithium ion intercalation-deintercalation reaction in different graphite-carbon active materials:

$6 \mathrm{C}+\mathrm{Li}^{+}+\mathrm{e}^{-} \leftrightarrow \mathrm{LiC}_{6}$

Morphology of one or another carbon material can affect quantitative indicators of $\mathrm{Li}^{+}$ion transport to some extent. However, at the microscopic level, for all these materials, reaction (5) actually occurs in the similar graphene layer and therefore, a scatter of 10 orders of magnitude seems unlikely.

There is no consistency in the reported descriptions of solid-state processes in a variety of layered cathode materials and for lithium batteries either. Moreover, even if $D$ value was measured in the same material by the same author using the same technique, its value changes by several orders of magnitude during the charge-discharge reaction. This indicates that $D$ is not the constant coefficient, as the diffusion theory (1) suggests.

In the attempt to justify the model of diffusion of protons (cations) in the solid phase of NHE, conducting polymers and some other systems with a solid-state reagent, some authors have cited the linear relationship existing between peak currents in voltammetric curves, $I_{\mathrm{m}}$, and square root of potential scan rate, $v^{-1 / 2}$, as the way of proof. This type of potentiodynamic dependence gives evidence for the presence of diffusion limitation and mathematically, it is substantiated only for liquid-state reactions but not solid-state ones.

Here, we will prove theoretically and experimentally that the linear dependence of $I_{\mathrm{m}} v s . v^{-1 / 2}$ and also linear dependence of potential difference formed between the anode and cathode peaks, $\Delta E_{\mathrm{m}}$ vs. $v^{-1 / 2}$, do not provide evidence for diffusion limitation in the reactions with a solid-state reagent, but rather indicate that there is a limited reserve capacity, $Q_{m}$ of the active material in such systems.

\section{Experimental}

To study a proton-conducting solid electrochemical system, p-chloranil was chosen. This sparingly soluble quinine shows linear current-voltage characteristic within almost the entire range of working potentials and an "ideal" reversibility [17]. P-chloranil was mixed with the battery graphite in a weight ratio of 1:1 and dispersed in a ball mill for 12 hours. Disc electrodes with diameter of $16 \mathrm{~mm}$ and thickness of about $1 \mathrm{~mm}$ were pressed from the resulting mixture under the pressure of $10^{5} \mathrm{~N} / \mathrm{m}^{2} .1 .5 \mathrm{M}$ sulfuric acid served as an electrolyte and an insoluble antraquinone electrode was the reference electrode.

The electrodes for lithium-ion batteries were produced by manual tape casting with different suspensions based on $\mathrm{N}$-methylpyrrolidone (NMP) on aluminum foil. The mixture with NMP contained 85 wt.\% active materials, 7 wt.\% carbon percolator and 8 wt.\% PVDF binder. As an active 
electrode material, $\mathrm{Li}_{4} \mathrm{Ti}_{5} \mathrm{O}_{12}$ (lithium titanate) from $\mathrm{MTI}$ Co. was chosen, while Timical $\mathrm{C} 65$ conductive carbon black was used as a conductive additive. Tape casting with $100 \mu \mathrm{m}$ gap was carried out by doctor's blade method. Dry electrodes with a thickness in the range 20-80 $\mu \mathrm{m}$ were calendared by a hot rolling calendaring machine from MTI. Then the electrodes were cut into $16 \mathrm{~mm}$ disk samples. The samples were further dried at $120^{\circ} \mathrm{C}$ and transferred to the argon filled MBraun glove box for assembling laboratory cells. Electrochemical tests were carried out using a coin-type cell (CR-2016) with lithium as reference and counter electrodes. The commercial separator (Celgard 2325) was placed between electrodes to ensure electrical insulation. $1 \mathrm{M}$ solution of $\mathrm{LiPF}_{6}$ in a mixture of ethylene carbonate (EC) and dimethyl carbonate (DMC) (weight ratio EC:DMC of 1:1) served as the electrolyte.

The electrochemical performance of materials was examined by cyclic voltammetry (CV) technique at different sweep rates (potentiodynamic curves). All electrochemical tests were performed using a multi-channel potentiostat/galvanostat VMP3 from Princeton Applied Research (UK).

\section{Results and discussion}

\section{Some theoretical approach}

The above mentioned approach towards postulating the diffusion model in a solid-state electrochemical system is not justified for the following few reasons:

1. Difference of applied potentials and corresponding electric field strength in the bulk solid phase and at the interfaces are the primary driving force for charge transfer in any solid-state electrochemical system. So, charge transfer in the solid state in any case occurs mainly by migration but not diffusion.

2. Typical diffusion processes in solid state is rather slow. For example, penetration of metallic ions into another metal on their direct "ideal" contact caused by a difference in concentrations, lasts for several months or even years (depending on nature of metals). Therefore, the time characteristic of diffusion processes is not comparable to the duration of electrode processes at discharge (charge) of batteries (tens of minutes, hours) and especially electrochemical capacitors (fractions of second, seconds).

3. In many real systems involving solid-state reagents, the process of diffusion in solid phase is absent as such. In our opinion, the main reason for the observed variance of $D$ values is that most of the solid-phase reactions involving charged particles like (3) and (4), proceed through migration mechanism, but not through diffusion mechanism. The driving force for these reactions is not a difference in concentrations, but a difference in potentials. Therefore, applying diffusion approach and diffusion parameters to these reactions is not sufficiently justified. In many cases, diffusion processes which rates in solid phase are typically very low, are negligible compared with migration processes in real systems with a solid-phase component. In such systems, a considerable contribution to the charge-transfer is made by the grain microstructure of the active material, its defectiveness and porosity, presence of interlayer water (for aqueous systems) or interlayer electrolyte (for non-aqueous systems), respectively.

Suppose that in a certain potential range, $\Delta E$, the voltammetric characteristic of an electrode is linear and the electrode can be characterized by an effective conductance, $G$, according to

$$
G=\frac{l}{\Delta E}
$$

where the change in potential is counted off the equilibrium potential of the electrode, $E^{0}$. 
The maximal charge-discharge capacity obtainable for a given electrode system during the linear sweep rate, $v$, can be calculated by the following equation:

$$
Q_{m}=\int_{0}^{\tau} G v t \mathrm{~d} t=\frac{1}{2} G v \tau^{2}
$$

In eq. (7), $t$ is time, and $\tau$ is duration of peak recording in the voltammetric curve.

On the other hand, the capacity can be calculated using the average current with taking into account the linearity section of the voltammetric curve as follows:

$$
Q_{m}=\frac{1}{2} l_{m} \tau
$$

Comparison of equations (7) and (8) and substitution of $\tau$ in the expression obtained from eq. (7), gives the following linear relationship between the peak current and the square root of potential scan rate:

$$
I_{\mathrm{m}}=\sqrt{2 G Q_{\mathrm{m}}} \sqrt{v}
$$

The model considered suggests a similar linear dependence of the difference between the anode and cathode peaks, $\Delta E_{\mathrm{m}}$, on the square root of potential scan rate

$$
\Delta E_{\mathrm{m}}=v \tau=\sqrt{\frac{2 Q_{\mathrm{m}}}{G}} \sqrt{v}
$$

These two equations were obtained without making any assumption about the mechanism of electrochemical process in the solid-state system (diffusion, migration, mixed or any other). Consequently, the linearity of the relationship between the peak current and the square root of potential scan rate cannot be presented as a sufficient evidence of the diffusion model.

Indeed, the linearity of voltammetric characteristic of the electrode within a certain section is only a priory assumption of the model considered, which is fairly justified regardless of the mechanism of electrochemical process. If such a linear section is relatively small, the voltammetric characteristic can be always presented as a sequence of linear sections using the patch-linear approximation, and derived conclusions can be applied to every individual section. Obviously, equations (9) and (10) will have a more complex nature.

Experimental cyclic voltammogramms of electrodes based on p-chloranil

Figure 1 shows potentiodynamic curves recorded at different scan rates for an electrode based on p-chloranil. It must be added here that bubbling of nitrogen helps to eliminate the possibly occurring side reaction of the corresponding hydroquinone oxidation by the oxygen dissolved in the acid:<smiles>O=C1C(Cl)=C(Cl)C(=O)C(Cl)=C1Cl</smiles>

As a result, the complete symmetry and closely spaced areas of anode and cathode peaks (charge and discharge capacities) which correspond to reaction (4) can be achieved (Figure 1).

Experimental data of peak currents and potential differences evaluated from Figure 1 and their statistical treatment for linear dependence on $v^{1 / 2}$ are shown in Figures 2 and 3, where linear dependences essentially cover the whole range of working potentials and are in full agreement with linear models defined by eqs. (9) and (10). At the same time, as mentioned above, the hydrogen 
transfer in such systems is not inherently diffusion, but probably proceeds by tunneling and depends on the distribution of electric field strength in the grain of quinone.

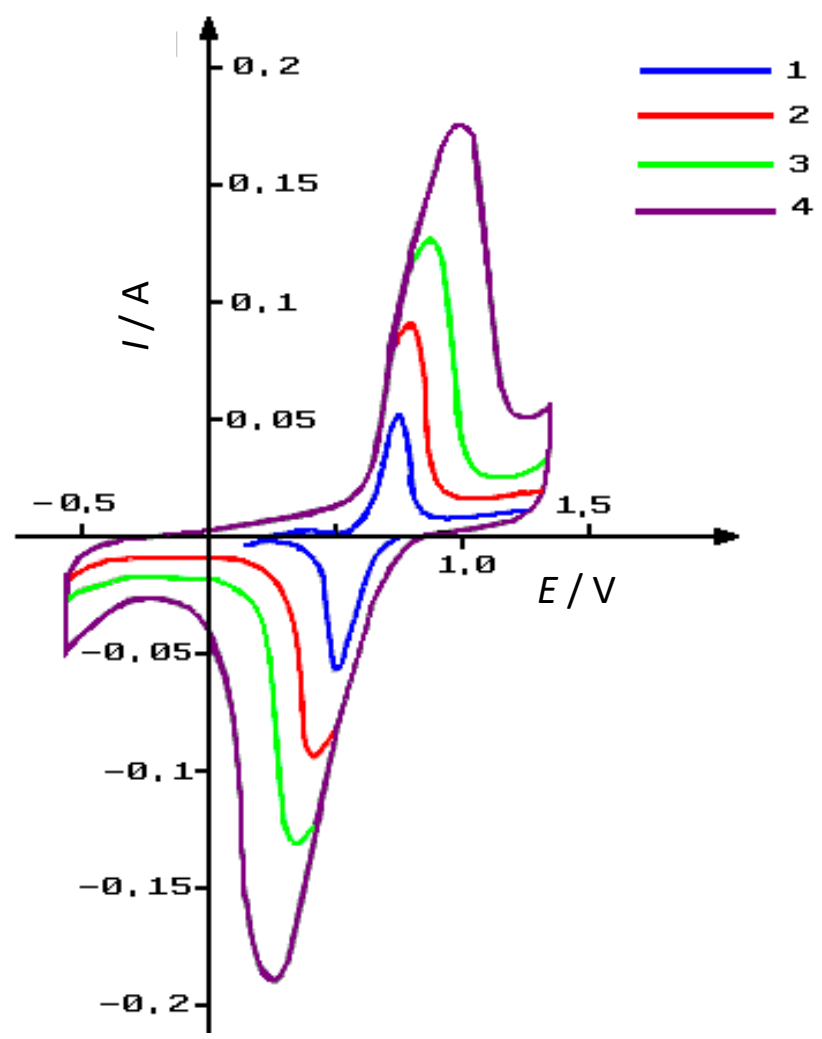

Figure 1. Cyclic voltammetric curves for electrode based on n-chloranil, recorded in $3 \mathrm{M} \mathrm{H}_{2} \mathrm{SO}_{4}$ with continuously bubbling nitrogen at different potential scan rates: 1) $0.10,2) 0.20,3) 0.40,4) 0.75 \mathrm{mV} \cdot \mathrm{s}^{-1}$

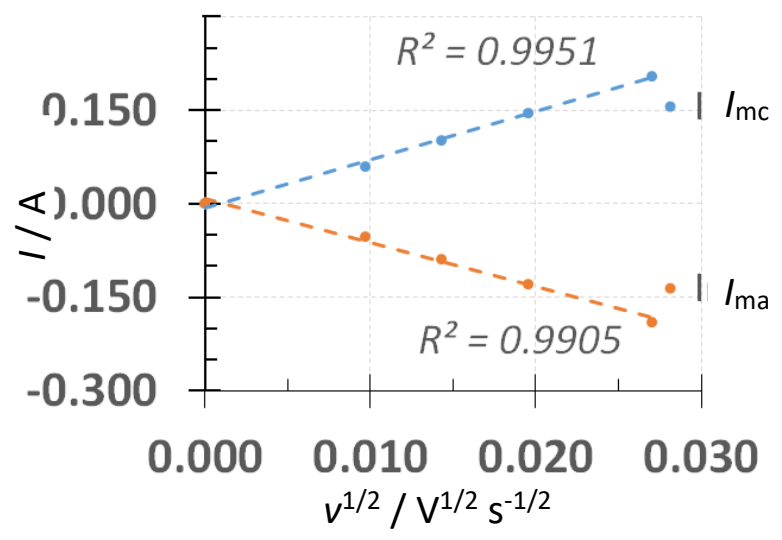

Figure 2. Anode $\left(I_{m a}\right)$ and cathode $\left(I_{m c}\right)$ peak currents (Fig. 1) vs. $v^{1 / 2}$

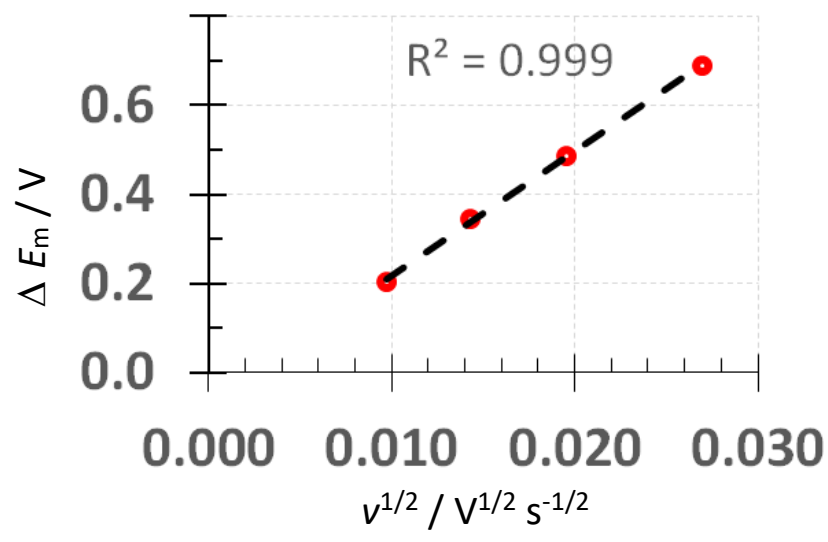

Figure 3. Potential difference $(\Delta \mathrm{E})$ between current peaks (Fig. 1) vs. $v^{1 / 2}$.

Experimental cyclic voltammograms of electrodes based on lithium titanate

Figure 4 shows cyclic voltammograms for a cell with a cathode based on lithium titanate at different scan rates. The curves represent intercalation-deintercalation of lithium cations into lithium titanate. The cathode part of the curve refers to the lithium deintercalation, while the anode part describes intercalation of lithium cation into lithium titanate. With increasing scan rate, the height and area of the peak increases. This is explained by the fact that the peak area divided by the scan rate gives the capacity of active material, which must be a constant. 


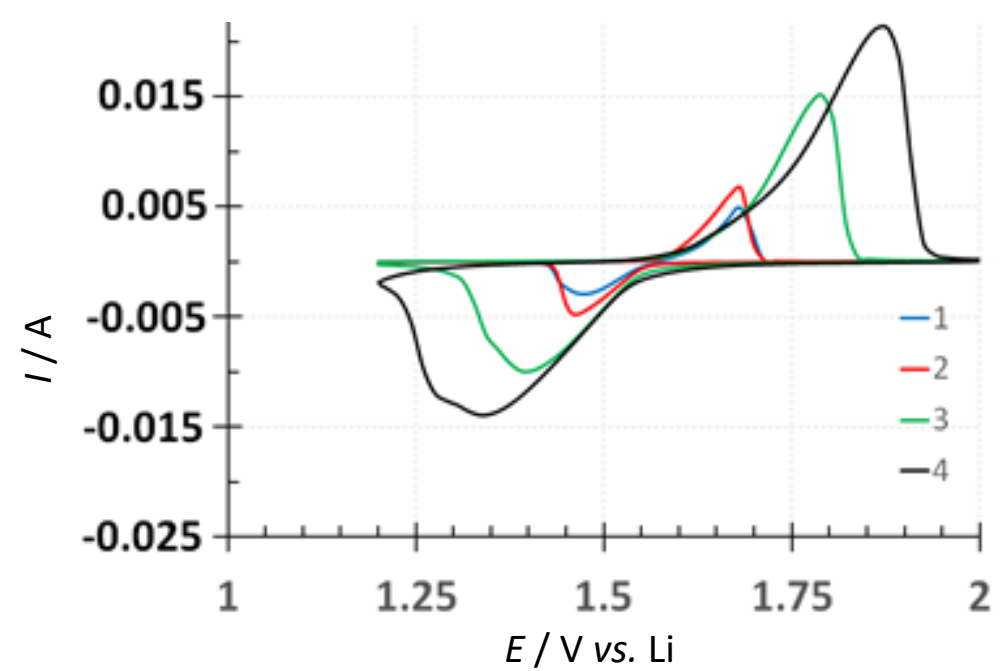

Figure 4. Cyclic voltammograms of lithium titanate electrode recorded at different scan rates:

1) 0.05 , 2) $0.10,3) 0.50,4) 1.00 \mathrm{mV} \cdot \mathrm{s}^{-1}$

Moreover, the anode peak shifts to the region of more negative potentials, whereas the respective cathode peak shifts toward more positive values. This is related to the fact that irreversibility of the process increases and manifests itself to a greater extent at high scan rates because lithium ions do not have enough time to deintercalate (intercalate) completely from the electrode. Otherwise, irreversibility is usually related to the limitation caused by diffusion of lithium cations in the solid phase of materials.

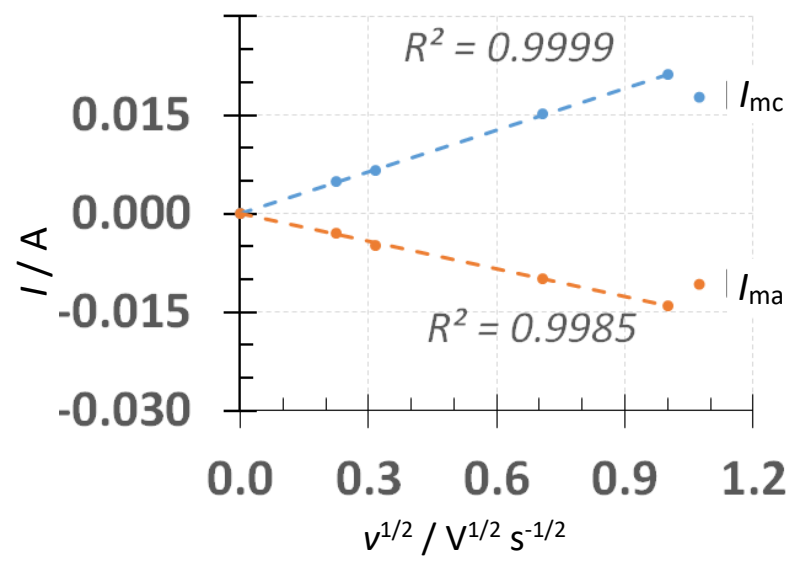

Figure 5. Anode $\left(I_{m a}\right)$ and cathode $\left(I_{m c}\right)$ peak currents (Fig. 4) vs. v $v^{1 / 2}$.

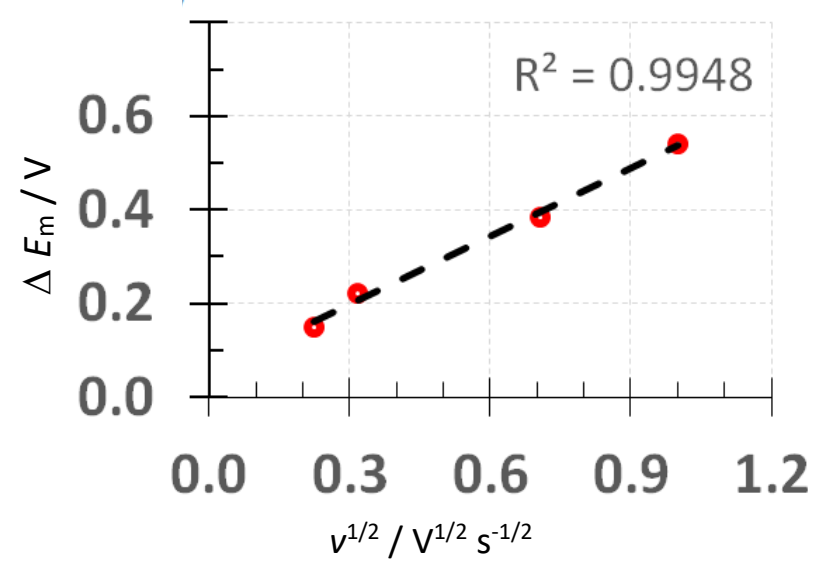

Figure 6. Potential difference $\left(\Delta \mathrm{E}_{m}\right)$ between current peaks (Fig. 4) vs. $v^{1 / 2}$

Quantitative evaluation of the diffusion coefficient is virtually impossible due to small change in concentration. Based on Figure 4, peak currents and potential differences between peaks against sq. root of potential sweep rate are shown in Figures 5 and 6 . The shift of peaks within the potential scale with increasing scan rate is observed in Figure 5 even at low potential sweep rates. This is why the assumption about the determining role of lithium ion diffusion in the solid state is not substantiated.

As seen in Figure 5, the peaks of the cathode and anode currents are linear functions of the square root of potential scan rate. Supporters of the diffusion model [18] usually suggest that the charge transfer through the interface is a fairly quick process and postulate that the rate limitation is only due to the diffusion of lithium within the electrode material. Then, by analyzing the slope of linear relation in Figure 5, the diffusion coefficient, $D$, could be calculated. In this case, apart from 
the above-mentioned contradictions, there arises a number of other problems. Examples are different $D$ values for the anode and cathode processes, or different $D$ values obtained with changing thicknesses of the active material.

The linear dependence of the cathode and anode peaks on the square root of potential scan rate (cf. Fig. 5), can also be interpreted by the change in ohmic resistance of the solid phase and the effect of lithium migration under the electric field. Recent successful usage of such a model for current distribution in a porous graphite electrode during the discharge process in lithium-ion battery or lithium-ion capacitor has been reported in [19].

\section{Conclusions}

A logical question put in this paper was: to which extent is it appropriate to use assumptions about diffusion and diffusion coefficient, $D$, for the processes being mainly of migration nature? Formally, $D$ can be connected to the mobility coefficient $\mu$ by the well-known Einstein equation

$$
D_{\text {eff }}=\frac{k T \mu}{e}
$$

where $k T$ is Boltzmann factor, $\mu$ is mobility coefficient and $e$ is electron charge.

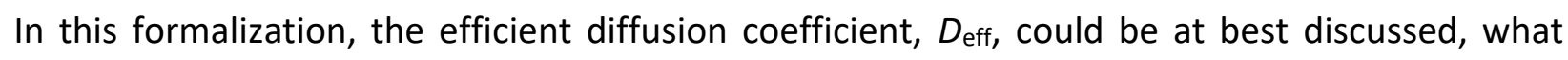
would not distort the ideas of charge-transfer migration mechanisms in the systems containing a solid-phase reagent.

In many real systems involving solid-state reagents, the process of diffusion in solid phase is absent as such. The existence of alternatives such as Grothuss, tunnel, and other fast mechanisms of cation migration in various solid-state systems often makes unnecessary considering of the longduration of non-competitive and energy-consuming diffusion process of heavy, charged particles in the solid phase.

In their attempt to justify the model of diffusion of protons (cations) in the solid phase, some authors have cited the linear relationship between peak currents, $I \mathrm{~m}$, in cyclic voltammetric curves and the square root of potential scan rate, $v^{-1 / 2}$, as the proof. In this work, we have shown that the existing linear dependences of $I_{\mathrm{m}}$ and also of potential difference between the anode and cathode peaks, $\Delta E_{\mathrm{m}}$, on $v^{-1 / 2}$ do not provide evidence for diffusion limitations in the reactions with a solidstate reagent, but rather that there is a limited stock of capacity, $Q_{m}$, of the active material in such systems.

\section{References}

[1] F. Brieuc, G. Dezanneau, M. Hayoun, H. Dammak, Solid State lonics 309 (2017) 187-191.

[2] B. Shruthi, B.J. Madhu, V. Bheema Raju, Journal of Energy Chemistry 25 (2016) 41-48.

[3] Q. Deyang, Electrochimica Acta 49 (2004) 657-665.

[4] P. M. Panchmatia, A. R. Armstrong, P. G. Brucec, M. S. Islam Physical Chemistry Chemical Physics 16 (2014) 21114-21118.

[5] W. Pfleging, P. Gotcu, Applied Science 9 (2019) 3588.

[6] J. H. Summerfield, C. N. Curtis, International Journal of Electrochemistry (2015), ID 496905, 10.1155/2015/496905.

[7] C.H. Hamann, A. Hamnett, W. Vielstich, in Electrochemistry, Wiley-VCH, Weinheim, New York, Chichester, Brisbane, Singapore, Toronto, 1998, p. 423.

[8] V. A. Volynskij, Ju. N. Chernyh, Elektrohimija [Russian Journal of Electrochemistry] 13 (1977) 11-16 (in Russian).

[9] V.A. Volynskii, PhD thesis, State University, Saratov, 1977, p. 127 (in Russian). 
[10] V. Z. Barsukov, L. N. Sagoyan, B. E. Rogoza, G. O. Yarkovoy, L. I. Zaslavskaya, in Batteries for Portable Application and Electric Vehicles, C.F. Holmes, A.R. Landgrebe (Eds.), Pennington, NJ, 1997, p. 823.

[11] V. Z. Barsukov, B. E. Rogoza, L. N. Sagoyan, $34^{\text {th }}$ ISE Meeting, Extended Abstracts, ISE, Erlangen, Germany, 1983, p. 112.

[12] V. Z. Barsukov, N. R. Meshcherjakova, L. N. Sagoyan, 36 th ISE Meeting, Extended Abstracts, ISE, Salamanka, Spain, 1985, p. 130.

[13] O. S. Ksenzhek, V. Z. Barsukov, V. V. Matveyev, $29^{\text {th }}$ ISE Meeting, Extended Abstracts, ISE, Budapest, Hungary, 1978, 2, p. 709.

[14] V. Z. Barsukov, V. V. Matveyev, $33^{\text {rd }}$ ISE Meeting, Extended Abstracts, ISE, Lyon, France, 1982, 2, p. 854.

[15] A. Churikov, A. Ivanishchev, A. Ushakov, Journal of Solid State Electrochemistry 18 (2013) 14251441.

[16] A. V. Churikov, Lecture at the Memorial Session devoted the memory of V.S. Bagotskii, Institute of Physical Chemistry and Electrochemistry, Moscow, Russia, $22^{\text {nd }}$ January 2013, 1-34 (in Russian).

[17] H. Alt, H. Binder, A. Kouhling, G. Sandstade, Electrochimica Acta 17 (1972) 873-887.

[18] J. Coelho, A. Pokle, S.-H. Park, N. McEvoy, N. C. Berner, G. S. Duesberg, V. Nicolosi, Scientific Reports 7 (2017) 7614.

[19] V. Barsukov, F. Langouche, V. Khomenko, I. Makyeyeva, O. Chernysh, F. Gauthy, Journal of Solid State Electrochemistry 19 (2015) 2723-2732.

(C)2020 by the authors; licensee IAPC, Zagreb, Croatia. This article is an open-access article distributed under the terms and conditions of the Creative Commons Attribution license (http://creativecommons. org/licenses/by/4.0/) 